\title{
FLARES IN LATE-TYPE STARS:
}

\author{
RADIO AND OPTICAL
}




\title{
Radio Emission from Flares in Single Late-type Stars
}

\author{
Arnold O. Benz \\ Institute of Astronomy, ETH-Zentrum, CH-8092 Zürich, Switzerland
}

\begin{abstract}
Radio observations provide the most direct information on nonthermal electrons in stellar flares and in the coronae of late-type stars. Radio emissions of single main-sequence $F, G$, and of many $K$ stars have recently been discovered, in addition to the well-known dwarf $M$ stars. Their long-duration radio flares with low circular polarization, slow variations and broad bandwidth can be attributed to gyrosynchrotron emission of mildly relativistic electrons. The same holds for the low-level ('quiescent') radio emission. On the other hand, highly polarized radio flares of $M$ stars have been interpreted by coherent emissions from loss-cone instabilities of magnetically trapped electrons. These conjectures are consistent with recent VLBI observations. The identification of the radio emission process allows to estimate the high-energy component of the flare and compare it to the total flare energy. The weakly polarized radio emission may serve as a proxy for hard X-ray signatures of relativistic electrons. The fraction of primary energy released into energetic electrons then appears to be large and similar to solar flares.
\end{abstract}

\section{Introduction}

Magnetic energy builds up by turbulent plasma motions in the interior of stars (where this phenomenon is called 'dynamo') and is transported into the corona by flux emergence and by footpoint motions of coronal magnetic fields. How is this magnetic energy and its associated currents reduced and released? Stellar flares in the coronae of protostars, interacting binary systems and rapidly rotating main-sequence stars present us with the challenge to develop models capable of interpreting the catastrophic annihilation of magnetic fields. The opportunity of stellar flares is to make the release process observable under a wide variety of physical conditions.

The Sun, a slowly rotating G2V star, is a convenient reference point. Solar hard X-rays (HXR) produced presumably by electron bremsstrahlung suggest that $20-40 \%$ of the energy in impulsive (regular) flares shows up initially in non-thermal electrons with energies in excess of $20 \mathrm{keV}$ (Duijveman et al. 1982, Strong et al. 1984). However, the estimate of energy partition is model dependent and still a matter of research. In reality it may depend on flare conditions and 
flare type. The non-thermal electrons are distributed in energy approximately according to a power-law reaching into the $\mathrm{GeV}$ range (e.g. Vilmer 1994, Petrosian et al. 1994). In stellar flares, where no HXR have yet been observed, radio emissions provide the most direct probes of non-thermal electrons. Observations in recent years have given ample evidence that stellar radio flare emissions originate from two groups of processes:

1. Gyrosynchrotron emission of mildly relativistic electrons. It is characterized by a broad bandwidth, low polarization and relatively slow variations in time.

2. Coherent emissions by instabilities of non-thermal electrons, appearing often, but not always, in a narrow frequency band with fast variations and high circular polarization.

The solar emissions demonstrate clearly that these general characteristics must not be blindly used, and the classification of observations is not straightforward. However, the distinction is essential for quantitative models since coherent emissions can be many orders of magnitude more efficient (e.g. Melrose 1986, Kuijpers 1989, Benz 1993).

It is beyond the scope of this brief review to describe stellar flare radio emissions in general. I shall focus my attention on single, late-type main-sequence stars that have a convective outer layer and a corona, and display activity similar to the Sun, although in some cases at a much higher level.

\section{Radio emissions from $F$ and $G$ stars}

Güdel et al. (1993) and Güdel (1992) have established a close linear relation between the quiescent thermal soft $\mathrm{X}$-ray emission and gyrosynchrotron radio emission of single, main-sequence $M$ and $K$ stars, respectively. The luminosities are related as $L_{x} \approx 10^{15.5} L_{r}$, where $L_{x}$ is in erg/s and $L_{r}$ in $\mathrm{erg} /(\mathrm{s} \mathrm{Hz})$ at $3.5-6$ $\mathrm{cm}$ wavelength. A similar ratio has been found in binary systems of the RS CVn type (Drake et al. 1989; Fox et al. 1994), Algols, BY Dra and pre-main-sequence T Tau stars (Güdel \& Benz 1993). Benz \& Güdel (1994) have measured about the same ratio also in solar flares, although with a significantly larger scatter. They have taken this to indicate that quiescent stellar coronae are heated by flare-like acceleration processes.

The radio/X-ray relation has recently inspired the search for radio emitting stars in regions of the Hertzsprung-Russell diagram where no non-thermal radio star has yet been known. Using the ROSAT survey as the guide, Güdel et al. $(1994,1995 \mathrm{a})$ have discovered quiescent radio emissions from five $G$ stars and four $F$ stars. To avoid errors, the stars have to be checked for cooler companions using the available spectrographic and astrometric information. Although in some cases the possibility of confusion with a low-mass companion remains, it has become unlikely to explain all the observations.

A remarkable example of these newly discovered radio sources is EK Dra (HD 129333), a GOV star and member of the Pleiades Moving Group (probable age 50-100 Myr). The quiescent radio emission is modulated by the $2.7 \mathrm{~d}$ optical 
rotation period, thus confirming for the first time a G-star origin of radio waves despite the known, distant M-star companion (Güdel et al. 1995b).

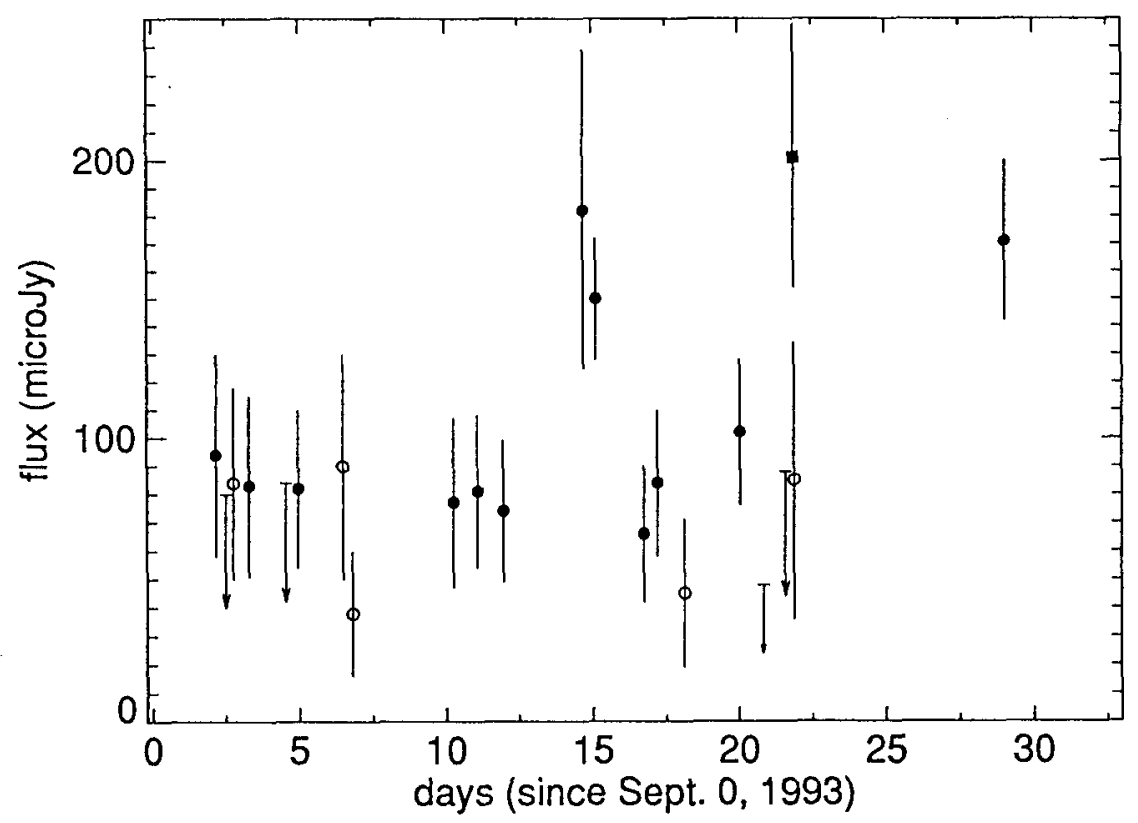

Fig. 1. Radio flux density of EK Dra observed in September-October 1993 by the VLA. Circles: $3.6 \mathrm{~cm}$; squares: $6 \mathrm{~cm}$; full symbols: $\gtrsim 2.5 \sigma$; open symbols: $\lesssim 2.5 \sigma$. Upper limits (arrows) refer to $2 \sigma$ at $3.6 \mathrm{~cm}$ (adapted from Güdel et al. 1995b).

The time line displayed in Fig. 1 shows occasional flares on top of the quiescent background. The radio flares have surprisingly long durations of 10-180 min and no detectable circular polarization $(<13 \%)$.

Figure 2 displays an X-ray time line of EK Dra (not simultaneous with Fig. 1). It shows a variety of time scales ranging from about 1.5 hours to gradual changes over days. Its modulation with a period of $2.7 \mathrm{~d}$ suggests again a G-star origin. The flares, like events $A$, and possibly $B$ and $C$, appear predominantly in the hard channel indicating high temperature. Events like A may occur at about the same rate as the radio flares apparent in Fig. 1.

The observation of weakly polarized flares on EK Dra are reminiscent of the radio emission observed from the G0V star $\chi^{1}$ Ori. The weakly polarized $(<30 \%)$ radiation reached a peak within 20 minutes and slowly decreased on a time scale of about one hour (Gary \& Linsky 1981, Linsky \& Gary 1983). As astrometric observations and infrared speckle interferometry have revealed an M4V companion star, the G-star origin has later been questioned (Linsky \& 


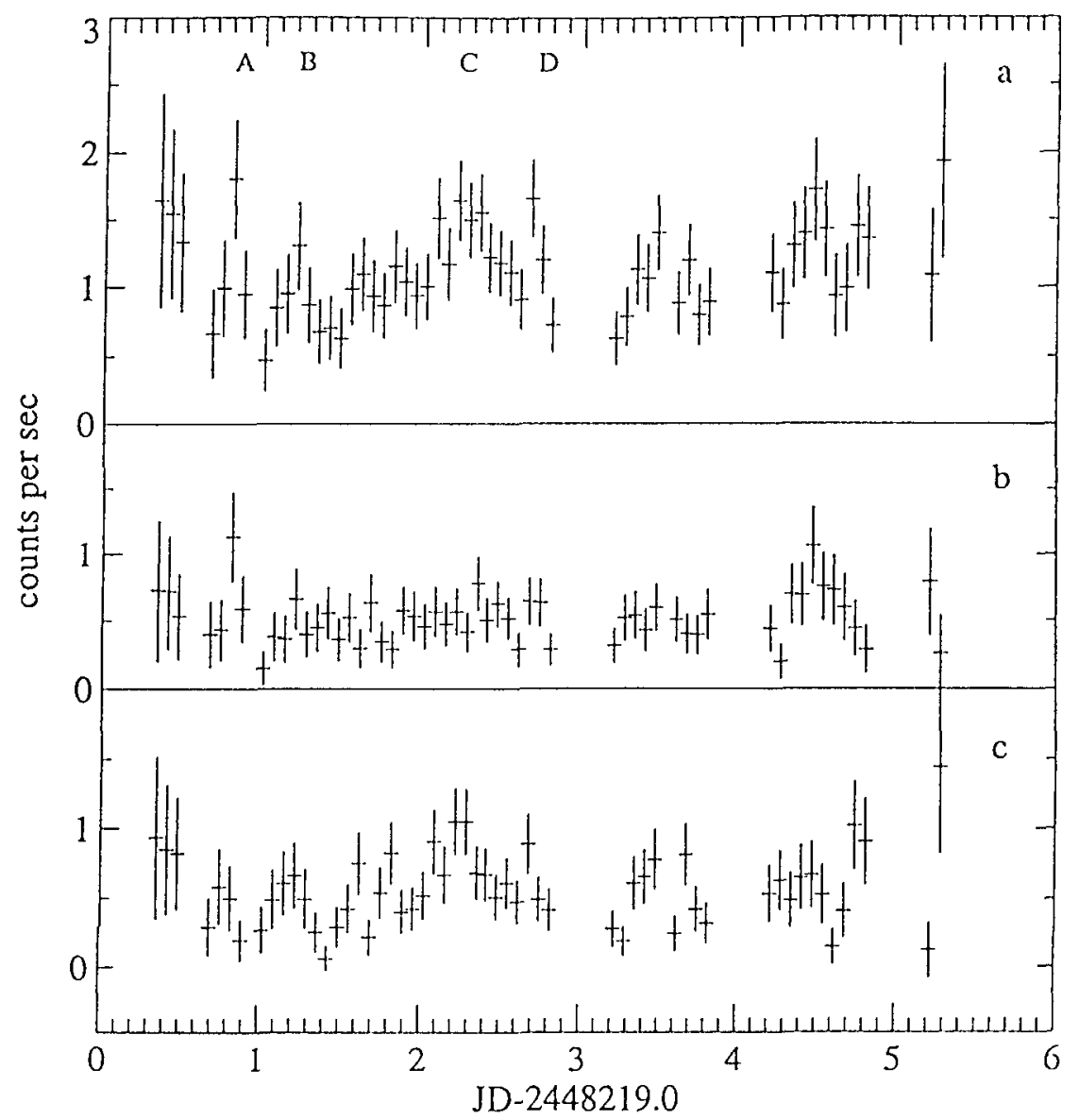

Fig. 2. Soft X-ray light curve of EK Dra observed by the ROSAT All-sky Survey from November 23-28, 1990, in Julian days with a sampling time of 96 minutes. a: all photons; b: hard channels (0.4-2.4 keV); c: soft channels $(0.1-0.4 \mathrm{keV})$. Flare-like events are labeled with letters $A$ to $D$ and are discussed in the text. (Adapted from Güdel et al. 1995b).

Gary 1983, Pallavicini et al. 1985), but not disproved. A flare possibly similar to large solar flares was detected on the F5 IV-V star Procyon, giving further evidence of ubiquitous coronal activity along the cool main sequence (Drake et al. 1993).

A flare similar in polarization (upper limit 16\%) and duration (about two hours), but an order of magnitude more luminous has recently been observed with high time resolution (Fig. 3 ). The flare radio luminosity has reached $2 \cdot 10^{16}$ erg $\mathrm{Hz}^{-1} \mathrm{~s}^{-1}$. The binarity of the G8V star is unknown. The similarities to the EK Dra flares and their difference to typical dMe-star flares suggest that the flare has occurred on the G star. 


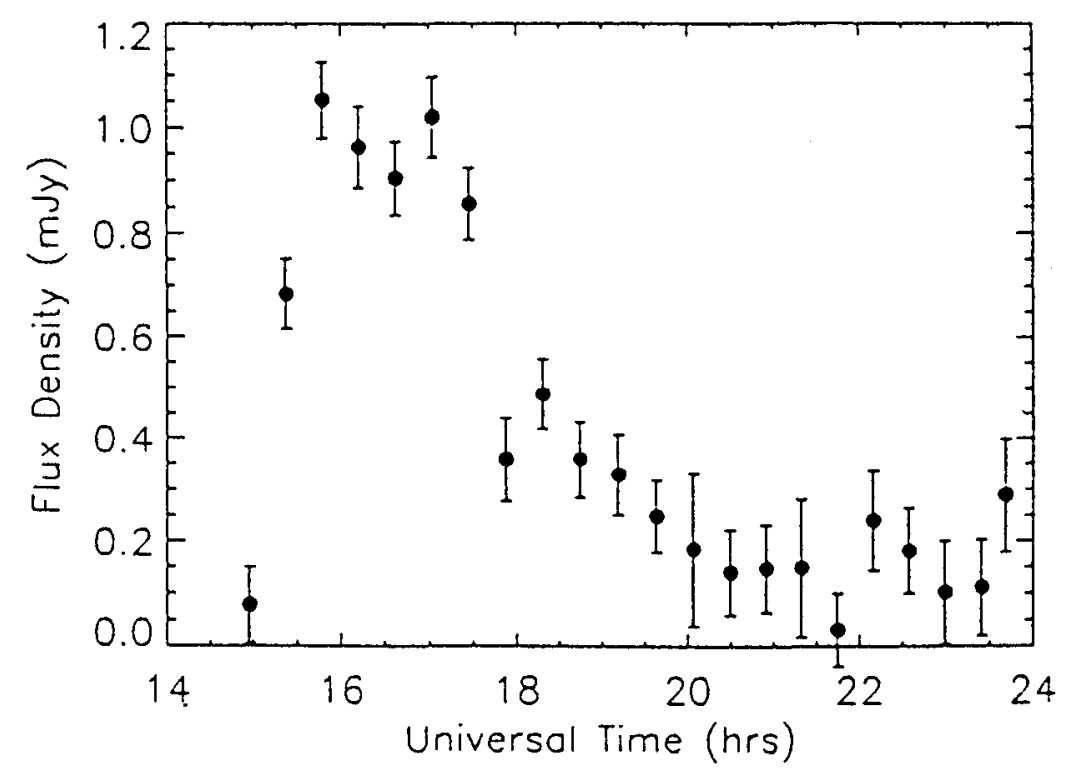

Fig. 3. Radio flare of the G8V star HII 1136 in the Pleiades observed on 1994 May 21 at $3.6 \mathrm{~cm}$ wavelength at the VLA (Lim \& White 1995).

Not many radio flares have yet been reported from main-sequence $K$ stars. Best known is the near or zero-age main-sequence $K 1$ star AB Dor. Longduration flares at $3.6 \mathrm{~cm}$ have been found with circular polarization of $13 \%$ and less (Slee et al. 1986). On the other hand, its radio flares at $6 \mathrm{~cm}$ wavelength lasting less than 1 hour have been reported to be highly circularly polarized (Lim et al. 1994). Furthermore, a prominent, weakly polarized $(<14 \%)$ flare has been registered in $3.6 \mathrm{~cm}$ observations of HD 147365, an F3IV-V star with a $\mathrm{K}$ type companion. The radio flare coincided spatially with the $\mathrm{K}$ star (Güdel et al. 1995a).

The vast literature on radio flares of dwarf $M$ stars has been reviewed by Bastian (1990) and Lang (1994). The reports on highly polarized flare emissions dominate (e.g. Gary et al. 1982, Lang et al. 1983, and many more). The degree of circular polarization in these flares is often, but not always, consistent with $100 \%$. Only few weakly polarized flares on dMe stars have been observed (e.g. Kundu et al. 1988, Güdel et al. 1993).

From the few observed radio flares of main-sequence $G$ and $K$ stars, it appears that low-polarization events dominate in $\mathrm{G}$ stars, coexist with high-polarization events in $K$ stars, and are a minority in $M$ stars. Whether these observations constitute a general trend has yet to be established. In particular, the frequency dependence of polarization should be investigated in the various stellar types. 


\section{VLBI and emission process}

The brightness temperature of a radio source can be used to test the proposed emission mechanism. Very long baseline interferometry (VLBI) of stellar flares can measure the radio diameter, $d$, or set an upper limit to it. The brightness temperature, defined as

$$
T_{b}=\frac{c^{2}}{\pi k_{B} \nu^{2}}\left(\frac{d}{D}\right)^{2} S,
$$

is then calculated from the observed frequency $\nu$, the source distance $D$, and from the flux density $S$ of one mode (e.g. left or right circular).

Gyrosynchrotron emission is an incoherent process, in which each particle radiates independently. Thus its brightness temperature is limited by the mean kinetic energy, $\langle\epsilon\rangle$, of the radiating electrons

$$
T_{\mathrm{syn}} \leq \frac{\langle\epsilon\rangle}{k_{B}} \quad[\mathrm{~K}]
$$

Equality is reached for optically thick emission. The synchrotron process encounters an absolute limit when the radiating electrons are cooled by inverse Compton scattering on their own emission. This 'inverse Compton catastrophe' sets in at about $10^{12} \mathrm{~K}$ (Kellermann \& Pauliny-Toth 1969). Assuming equipartition of the energy densities between the radiating particles and the magnetic field and using the magnetic field estimate from the spectral peak caused by synchrotron self-absorption, Readhead (1994) has derived an upper limit on the synchrotron brightness temperature of a few times $10^{11} \mathrm{~K}$ (depending on the spectral index).

Since coherent emission is caused by non-thermal, but not necessarily relativistic particles, the inverse Compton effect can usually be neglected for this type of emission. Consequently, the catastrophe and equipartition upper limits do not apply.

Most VLBI studies of flares have concentrated on bright RS CVn type binaries. A weakly polarized flare on the RS CVn binary HR 1099 (V711 Tau) has been reported by Trigilio et al. (1993). The initial brightness temperature of the flare at $6 \mathrm{~cm}$ was $4.60( \pm 1.16) \cdot 10^{9} \mathrm{~K}$. It decreased slowly and continuously to $1.72( \pm 0.67) \cdot 10^{9} \mathrm{~K}$ after 2.5 hours. These values are consistent with the interpretation by gyrosynchrotron emission of mildly relativistic electrons. Using Eq. (2), their initial mean energy is derived to be $\geq 400 \mathrm{keV}$.

Coming back to single main-sequence stars, a highly polarized flare with a very structured time profile has been observed in VLBI at $18 \mathrm{~cm}$ on the single dMe star EQ Peg by Benz et al. (1995). The flux densities measured by VLBI baselines do not deviate from the values observed by the VLA. It indicates that the radio source has not been resolved and must be smaller than 0.5 mas (or $5 \cdot 10^{10} \mathrm{~cm}$ ). A brightness temperature $>4 \cdot 10^{10} \mathrm{~K}$ results, which is below the limit of the equipartition value. The resolution was not sufficient to discard synchrotron emission, but puts the extreme requirement, that the mean electron 
energy would have to exceed $4 \mathrm{MeV}$. This exceeds the solar values by two orders of magnitude and is not a likely interpretation.

\section{Radio/X-ray ratio of G-star flares}

The detection of weakly polarized flares in single main-sequence $G$ and $K$ stars opens the prospect of estimating the flare energy released into energetic electrons. Direct energy calculations from synchrotron emissions are plagued by many free model parameters, such as trapping time, aspect angle, anisotropy, to name just a few. Instead I suggest a heuristic approach based on a comparison with solar flares.

The flux density of a typical radio flare of EK Dra is about $100 \mu \mathrm{Jy}$ (Fig. 1). This may be compared to the $\mathrm{X}$-ray flux of the largest flare in a comparable time period, flare $A$ in Fig. 2. It peaks at about $1 \mathrm{ct} / \mathrm{s}$ above background, corresponding to $7 \cdot 10^{29} \mathrm{erg} / \mathrm{s}$ at the distance of EK Dra if the same standard ROSAT (PSPC) conversion factor is adopted.

The resulting ratio of the two luminosities, $L_{x} / L_{\tau} \approx 10^{15.8} \mathrm{~Hz}$. It is close to the value $10^{15.5} \mathrm{~Hz}$ derived by Benz \& Güdel (1994) for solar flares (impulsive and gradual). The observed X-ray flare on EK Dra produced an energy of $5 \cdot 10^{33}$ erg in thermal $\mathrm{X}$-rays, about two orders of magnitude more than the largest solar flare; and the non-thermal radio flux was found at a proportionally higher level. It strongly suggests that the energy partition between thermal and non-thermal components is similar in solar and stellar flares.

\section{Conclusions}

$\mathrm{F}, \mathrm{G}$, and $\mathrm{K}$ main-sequence single stars have been discovered in radio emission in considerable numbers. The few radio flares that have yet been observed seem to be different from the typical flares on dMe stars:

- They tend to be weakly polarized and seem to be caused by synchrotron emission.

- They last longer, but are less frequent.

- Since their emission process is probably incoherent and thus less efficient, more non-thermal energy is needed to produce the same flux. Thus they may represent larger flares.

These trends need to be confirmed by more observations.

Multiwavelength flare observations of dMe stars have frequently resulted in the disappointing statement that there was no radio enhancement during an optical or X-ray flare, or that no optical or X-ray counterpart was observed during a radio flare (e.g. Karpen et al. 1977, Kahler et al. 1982). There is a strong suspicion that in all such cases the radio telescope was not sufficiently sensitive to detect the weakly polarized synchrotron emission or, respectively, the radio emission was strongly polarized, indicating highly efficient coherent emission by a possibly minor flare. 
The radio emission of weakly polarized flares on a young G-dwarf seems to follow the same radio/X-ray relation for peak values as solar flares. This suggests that the stellar flares are comparable in acceleration efficiency for energetic electrons. It further suggests that the weakly polarized stellar radio flare emission is produced in a similar way. Emission models adapted from solar flares thus provide quantitative information on these electrons. The weakly polarized radio emission may serve as a proxy of hard X-ray bremsstrahlung of relativistic electrons used successfully to study the energetics of solar flares.

Acknowledgements: I thank Manuel Güdel for helpful discussions. This work has been supported by the Swiss National Science Foundation (grant 20-040336.94).

\section{References}

Bastian T.S., 1990, Solar Phys. 130, 265

Benz A.O., 1993, Plasma Astrophysics, Kluwer, Dordrecht, Holland

Benz A.O., Alef W., Güdel M., 1995, A\&A, in press

Benz A.O., Güdel M., 1994, A\&A 285, 621

Drake S.A., Simon T., Linsky J.L., 1989, ApJS 71, 905

Drake S.A., Simon T., Brown A., 1993, ApJ 406, 247

Duijveman A., Hoyng P., Machado M., 1982, Solar Phys. 81, 137

Fox D.C. et al., 1994, A\&A 284, 91

Gary D.F., Linsky J.L., 1981, ApJ 250, 284

Gary D.F., Linsky J.L., Dulk G.A., 1982, ApJ 263, L79

Güdel M., 1992, A\&A 264, L31

Güdel M., Benz A.O., 1993, ApJ 405, L63

Güdel M., Schmitt J.H.M.M., Bookbinder J.A., Fleming T., 1993, ApJ 415, 236

Güdel M., Schmitt J.H.M.M., Benz A.O., 1994, Science 265, 933

Güdel M., Schmitt J.H.M.M., Benz A.O., 1995a, A\&A 293, L49

Güdel M., Schmitt J.H.M.M., Benz A.O., Elias N.M., 1995b, A\&A, in press

Kahler S. et al., 1982, ApJ 252, 239

Karpen J. et al., 1977, ApJ 216, 479

Kellermann I.I., Pauliny-Toth I.I.K., 1969, ApJ 155, L71

Kuijpers J., 1989, Solar Phys. 121, 163

Kundu M.R., Pallavicini R., White S.M., Jackson P.D., 1988, A\&A 195, 159

Lang K.R., 1994, ApJSS 90, 753

Lang K.R., Bookbinder J., Golub L., Davis M.M., 1983, ApJ 272, L15

Lim J., White S.M., Nelson G.J., Benz A.O., 1994, ApJ 430, 332

Lim J., White S.M., 1995, ApJ, in press

Linsky J.L., Gary D., 1983, ApJ 274, 776

Melrose D.B., 1986, Instabilities in space and laboratory plasmas, Cambridge Univ. Press, Cambridge

Pallavicini R., Willson R.F., Lang K.R., 1985, A\&A 149, 95

Petrosian V., McTiernan J.M., Marschhäuser H., 1994, ApJ 434, 747

Readhead A.C., 1994, ApJ 426, 51

Slee O.B. et al., 1986, Proc. ASA 6, 312

Strong K.T. et al., 1984, Solar Phys. 91, 321

Trigilio C., Umana G., Migenes V., 1993, MNRAS 260, 903

Vilmer N., 1994, ApJS 90, 611 
M. Shapiro: Dr. Benz has told us that coronal heating is related to the acceleration of electrons in active stars. Is it not likely that energetic protons also contribute significantly to the heating?

A.O. Benz: This is certainly possible. Some acceleration processes, such as shock acceleration or some current instabilities, put more energy into protons that electrons. Such models have not received much support from solar observations. Nevertheless, protons remain the big unknowns in both solar and stellar flares.

R.E. Gershberg: Did you estimate a probable contribution of the total stellar population radiation at microwaves in the galactic and cosmological background? A.O. Benz: The quiescent flare star radio emission constitutes a background of about $10^{-5}$ of the cosmological background at $6 \mathrm{~cm}$. In addition, it usually decreases with decreasing wavelength, contrary to the cosmological radiation that has a maximum around $1 \mathrm{~mm}$.

I. Pustylnik: You have shown the beautiful pictures of radio isophotes for the two binaries EK Dra and HR 963. Do you see these objects as a single radio source with isophotes concentrated more to one or the other star, or as a binary radio source?

A.O. Benz: In the case of HR 963, the radio source is consistent with the optical position of the F7 IV star within the positional accuracies. There is no indication of plasma physical interactions with the $\mathrm{G} 7 \mathrm{~V}$ companion at a distance of $1.8 \times 10^{15} \mathrm{~cm}$. EK Dra is probable a single star. In any case, the rotational modulation of the radio emission is consistet with a G0V star. 